This document is published in:

Cabestany, J. et al. (eds.), 2011. Advances in Computational Intelligence: 11th International Work-Conference on Artificial Neural Networks, IWANN 2011, Torremolinos-Málaga, Spain, June 8-10, 2011, Proceedings, Part II. (Lecture Notes in Computer Science, 6692), Springer, pp. 621-628.

DOI: 10.1007/978-3-642-21498-1_78

(C) 2011 Springer-Verlag Berlin Heidelberg 


\title{
Research Opportunities in Contextualized Fusion Systems. The Harbor Surveillance Case
}

\author{
Jesus Garcia ${ }^{1}$, José M. Molina ${ }^{1}$, Tarunraj Singh ${ }^{2}$, John Crassidis ${ }^{2}$, and James Llinas ${ }^{2}$ \\ ${ }^{1}$ GIAA, Universidad Carlos III de Madrid. Av. Univ. Carlos III, 22, Colmenarejo, Spain \\ \{jesus.garcia, josemanuel.molina\} @uc3m.es \\ ${ }^{2}$ CMIF, State University of New York at Buffalo, Bell Hall 313. Buffalo, NY 14260, USA \\ \{llinas, tsingh, sudit, crassidis\}@buffalo.edu
}

\begin{abstract}
The design of modern Information Fusion (IF) systems involves a complex process to achieve the requirements in the selected applications, especially in domains with a high degree of customization. In general, an advanced fusion system is required to show robust, context-sensitive behavior and efficient performance in real time. It is necessary to exploit all potentially relevant sensor and contextual information in the most appropriate way. Among modern applications for IF technology is the case of surveillance of complex harbor environments that are comprised of large numbers of surface vessels, high-value and dangerous facilities, and many people. The particular conditions and open needs in the harbor scenario are reviewed in this paper, highlighting research opportunities to explore in the development of fusion systems in this area.
\end{abstract}

Keywords: Harbor surveillance, Sensor Fusion, Context Representation, Situation Analysis.

\section{Introduction}

The continuous development of new applications of Information Fusion (IF) has increased the research interest in applicable techniques to extend capabilities of current ones. Nowadays, fusion systems are oriented to the integration of all types of sensor data and available information in databases, knowledge experience, contextual information, user mission, etc, in order to generate value-adding fused estimates of the conditions of interest in dynamically-changing situations. In particular, among the modern research challenges for IF process and algorithm design, has been the design and development of techniques to exploit contextual information, which is any kind of information which helps better characterize the situation/state. Contextual information can aid both the formulation of an improved state estimate and the interpretation of a computed estimate. However, the incorporation of contextual information adds complexity and demands new, hybrid techniques be developed in order to use different sources of information in an integrated reasoning process.

A current need is the study of novel methods to enhance current IF systems, applying adaptive paradigms to the management of processes, sensors and other available knowledge. Usually fusion systems are described according to JDL fusion model. Recent articles suggesting revisions and extensions of this model point to key aspects 
in real applications [1],[2] such as evaluation and quality-control processes to increase reliability, the adaptation mechanisms to maximize the output value in the application, the need for and exploitation of an ontologically-based approach, or the role of distributed IF.

The two general objectives highlighted in this work are (i) exploring ways of modeling contextual information in the fusion process, looking for possible general formalizations, and (ii) design and development methods for adapting the data processing systems based on the fusion quality, context and requirements imposed by the application. The research in these areas will be focused in studying IF technology applications to maritime surveillance, with a particular emphasis on the harbor/port surveillance problem.

\section{The Maritime Scenario. Requirements and Research Needs}

The selected application, maritime surveillance, is a high-priority aspect for national and international security programs, especially in the coastal waters, borders and ports. The surveillance in these zones faces challenging problems to analyze such as terrorist threats, maritime and ecological accidents, illegal immigration, illegal fishing, drug trafficking, etc. Therefore, the research in maritime surveillance is mainly promoted by state agencies such as NATO, who supports to research on Maritime Surveillance, and national programs like Hawkeye or Centurion 0 projects in the United States to prevent threats in ports. In the European Maritime Policy "Blue Paper" [4], the European Commission states a general target of European surveillance network, composed of "interoperable surveillance system" for maritime safety, protection of the marine environment, fisheries control, control of external borders and other law enforcement activities.

\subsection{Sensor Fusion for Maritime Surveillance}

In order to achieve the required level of quality in maritime surveillance, it is necessary to use a heterogeneous network of sensors and a global multi-sensor tracking and fusion infrastructure capable of processing the data. The system must determine potential threatening objects within a scene containing a complex, moving background, minimizing the errors (both false positives and negatives), and exploiting scenerelated knowledge to exploit the sensor data with maximum accuracy.

There are varied technologies for detection and location (coastal radar, video cameras, IR, automatic identification system, etc), but none of them alone are able to ensure reliable surveillance for handling complex scenarios. For example, high resolution coastal radar technology is effective with high accuracy and availability, but usually presents difficulties which make it necessary to supplement with cooperative location technologies. Radar can have problems such as occlusions, shadows, clutter, etc., and difficulty detecting small boats, because they are very small with low detectablity (for instance small inflatable boats in trafficking activities or skiffs in piracy, both with poor radar returns). Automatic Identification System (AIS) technology can provide situational awareness with positive identification of approaching vessels, but they are obviously insufficient on their own, because of needed cooperation, and 
occasional presence of anomalous data, losses in coverage etc. Therefore it is a usual situation to seek help of additional sensor sources such as computer vision systems to improve the detectability of all type of targets. The fusion system must take into account the characteristics of all data sources. Research of appropriate architectures and algorithms for multi-sensor fusion in this environment is needed, especially with large and heterogeneous areas and high density spaces with large numbers of very diverse tracked objects (tankers, ferries, sailboats, inflatable boats, etc.).

\subsection{The Harbor Scenario as a Highly Contextualized Case}

The harbor is one of the most complex maritime scenarios. The surveillance system in this area must analyze the situation to monitor and control entry to ports and land waterways using the available sensors and other sources with very specific information. The concerns of surveillance system are safety and efficiency in traffic operations, with additional considerations regarding the operation of oil and gas stations. Besides, the representation of situation will allow provide the pilots with aids such as navigation in port and docking assistance (which can be mandatory depending on vessels type and harbor configuration).

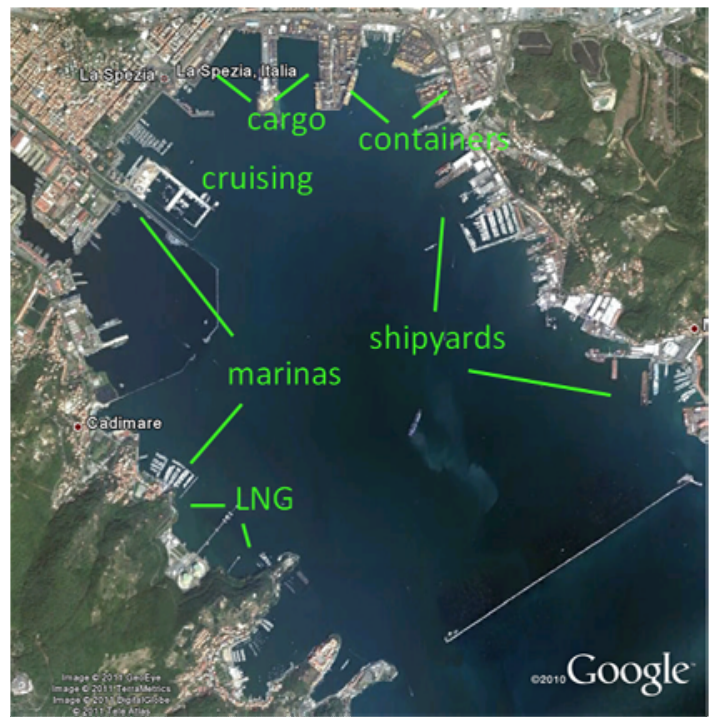

Fig. 1. Configuration of harbor La Spezia, Italy

An example of port configuration is shown in Fig. 1. We have selected Port of La Spezia [3] for illustration because it clearly illustrates the complex portfolio of activities that can take place in large ports. We can appreciate the very diverse nature of operations carried out in a big harbor like La Spezia, in Italy. In a reduced area there is coexistence of very diverse operations: cargo containers traffic, routes for related with Liquid Natural Gas re-gasification terminal (LNG), passenger operations, recreational boats in marinas, defense, etc. 
There are clear indications for pre-planned ship mooring arrangements, approachspeed monitors and mooring strain sensors to facilitate vessel arrival and support safe mooring operations to eliminate damage to facilities. Some examples of mandatory and suggested routes and traffic indications in this specific site are indicated below (depicted in Fig. 2):

\section{Outer channels:}

- Merchant ships must follow a "safe speed", taking care of traffic conditions and circumstances at any moment, and avoiding the production of waves that can cause troubles to small boats/vessels which, in particular during summertime, are used to sail in the area and along the coast.

- Some zones are considered dangerous for maritime traffic due to military exercises. Three compulsory tracks for landing at la Spezia Port are defined

\section{Inner channels:}

- Towed ships coming in or out of the inner channels have precedence over all other ships or boats inside inner channels; merchant ships cannot exceed six knots in speed, except extraordinary circumstances imposed by rigging demands.

- When the vessel meets vessels sailing in the opposite direction and/or when are closed to berthed vessels, they have to decrease their speed if possible.

- The connection between Punta San Bartolomeo and the white light of Molo Lagora it is possible the transit of vessel with at most 30' deep draft.

- In the southern the connection between the top of Enel wharf and the root of Molo Lagora it is possible the transit of vessel with at most 27' deep draft.

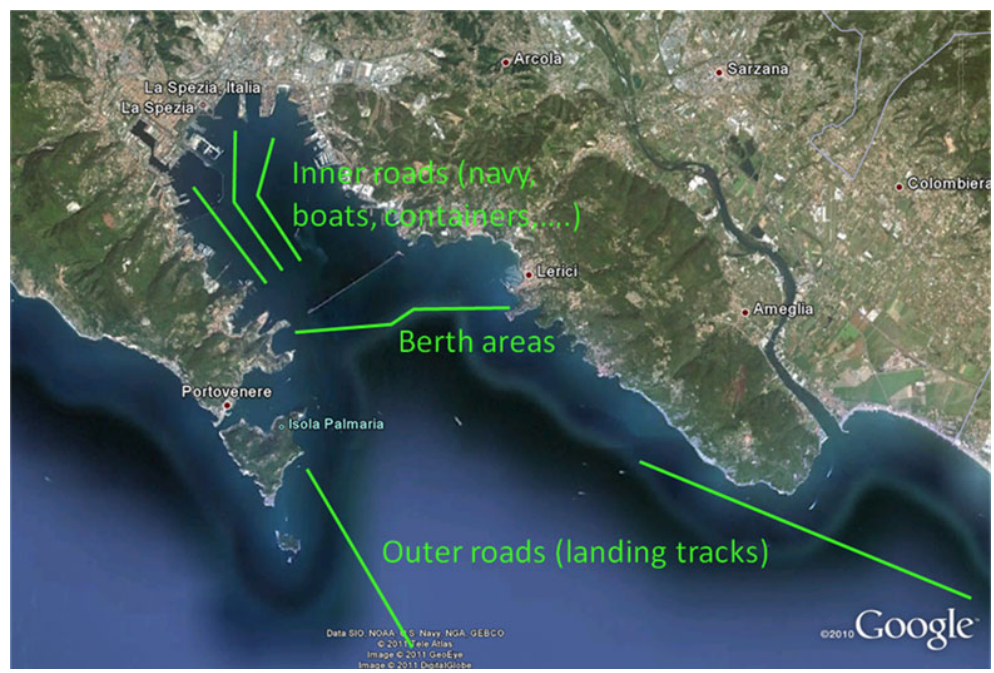

Fig. 2. Routes for traffic of the harbor in La Spezia, Italy

So there is much information related with regulations and predefined behavior in the harbor area, information that should be used to characterize the situation, interpret the sensor data and focus the analysis in the expected normal operations. The process of 
assessing situations and threats requires monitoring not only critical harbor facilities but also linked coastal areas, sea surface, underwater, etc. Some of the issues defining this scenario are:

- $\quad$ Large number of vessels ranging from small recreational sailboats, tug boats, jet skis, to commercial vessels.

- Detection difficulties such as clutter and low resolution

- Tracking requires good data association for a multi-sensor tracking scenario

- Threats and potential conflicts for traffic operation (such as lack of adequate separation) must be detected.

\section{General Approach for Context-Based Adaptive Fusion System}

Complexity in maritime and harbor scenarios calls for designing advanced fusion systems capable of processing all available sensor data and information to support the multiple decision makers at different levels. Figure 3 shows a general approach to context-based adaptive fusion. It follows the structure proposed by Llinas [2] for a general framework of robust information fusion systems, where multiple processes work in parallel to achieve context-sensitive and adaptive behavior.

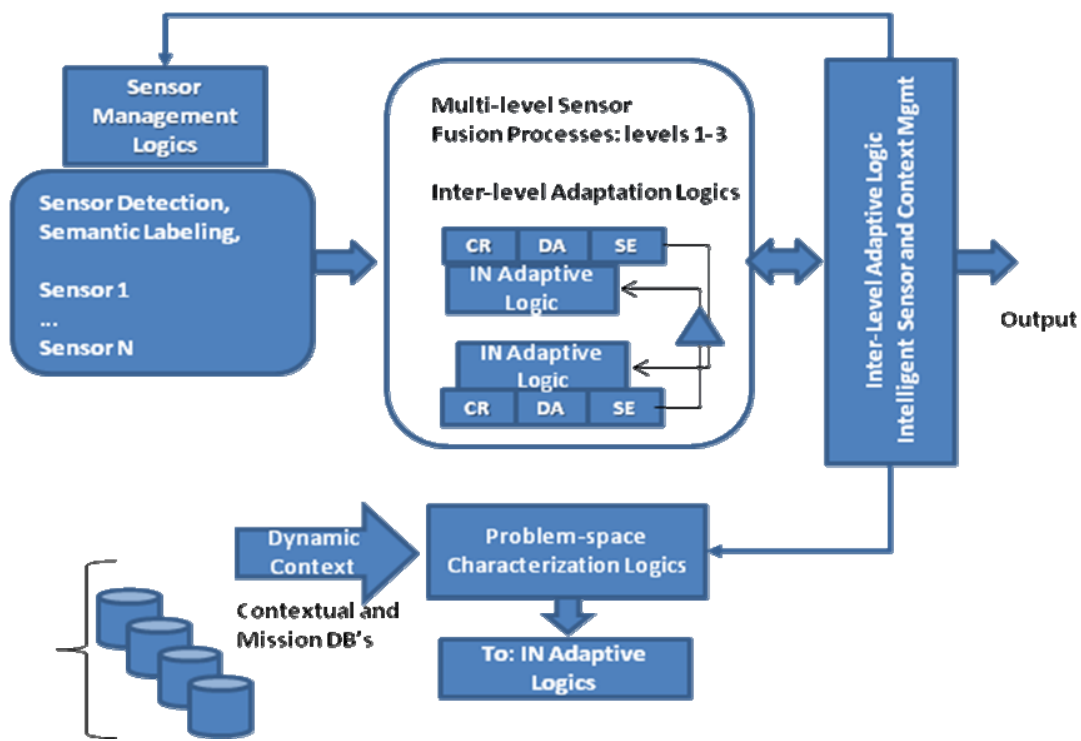

Fig. 3. Overall architecture of the general context reasoning environment [2]

Here, the core with fusion processes contains levels 1-3, and the external adaptation process belongs to level 4. Every IF process in JDL is abstracted in the three functions of Common Referencing (CR), Data Association (DA, and State Estimation (SE). Several aspects are considered with respect to a non-adaptive conventional fusion system: 
- A function module called Problem Space Characterization Logic is in charge of adaptively managing the system. Making use of contextual knowledge and a library of alternative algorithms, an intelligent algorithm manager can terminate and invoke the best algorithm for the current problem-space condition. Although advanced fusion algorithms work close to optimality, they usually operate under limited conditions (assumed models, sensor performance, information consistency, etc.). The combination of different intelligent techniques is a possibility to overcome limitations of individual techniques and available implementation of fusion algorithms, with the aim of adapting the fusion system performance to the requirements, and to different situations.

- Contextual information has an essential role, which feeds this knowledge base and adaptation logic. The adaptation of data processing algorithms (extensible to sensor management when they have control parameters, such as coverage, refresh cycle, etc.) needs a model of interrelation between all aspects of fusion.

\section{Research Areas in Harbor Scenario Applications}

Finally, among the open challenges to develop advanced fusion systems in the context-dependent situation as a harbor case, we indicate three specific research challenges as immediate steps: context-aided tracking systems, situation analysis and coordinated action detection.

\subsection{Contextualized Sensor Fusion}

The area of contextually-enhanced multi-sensor, multi-object tracking is very important in complex scenarios where classical techniques alone (typically based solely on observational data) are not enough to model the objects behavior and dynamics of situation. The contextual information about the environment allows a more accurate interpretation of sensor data and the adaptation/optimization of system performance. Examples of sources of contextual knowledge have been mentioned before: geometrical configuration of harbor, moving lines and mooring areas, speed limit, types of vessel and associated priorities, etc.

Depending on the nature of context information, different strategies are appropriate. The a priori known static context can be used to determine the representation and constraints to be taken into account in the inference processes. For example, assign a detected object with its category of vehicle, whose constraints are known, can be used to refine its dynamic model. In this way, in [5], [6], fusion parameters are adapted depending on regions (sea, ocean, regions of high clutter density, etc.). In recent works related in port areas [7], the limitations derived from channel depth, limited areas, etc. are used to characterize the "trafficability" and refine the prediction models. Knowledge of dynamic context also enables algorithms to interpret the data received [8]. A dynamic representation of contextual information, inferred with the help of other fusion levels and their contextual information can be used to enhance the sensor data processing. An example can be the description of multi-object situations such as vessels in coordinated motion according to traffic movement protocols, expected maneuvers, knowledge of relationships between entities, active pilotage/towage operations among vessels and tug boats, etc. 


\subsection{Situation Analysis}

The inference of suspicious object behavior is one of the main objectives of fusion systems, in order to focus the operator attention. Many factors can be used to characterize the situation: object classes, speeds, relative positions (with respect to allowed zones according to its class, etc.). The surveillance system must decide which events are anomalous, to recognize the situation of mobile elements with respect to the static elements in the scene. Contextual information can clearly help understand whether certain behaviors are anomalous or not.

Rule-based systems can be configured to identify situations that are out of the norm and provide alarms, such as the presence of ships unidentifiable or vessels approaching unsafe waters. The definition of normal behavior accordingly to maritime/harbor rules should be formalized, with the possibility of ontology formalisms to represent and include reasoning mechanisms at different levels [9].

\subsection{Collective Behavior Analysis}

An important challenge for situation analysis in the harbor and maritime scenarios is the capability to detect or anticipate collective behavior that may represent threats. An example is a coordinated attack of several boats against a certain target (big ship, land facility, etc.). Contextual information can help in such IF-based estimation techniques by providing inputs that define known coordinated vessel activities such as planned tugboat-ship operations, coordinated derding operations, etc.

Group behavior recognition would be based on features referred to several objects. A possibility is a trajectory-based approach in which multi-agent action recognition involves the compact representation and modeling of actions and interactions, as well as their logical and temporal relations. This type of approach has been used in works related with robotics and sports analysis [10]; most of them divide the collective activity into individual actions. The application of these approaches to this problem would need in the first step to have available the individual trajectories of all objects, which depends on the sensor resolution and capability of tracking systems.

\section{Conclusion}

We have presented some important open issues for modern information fusion systems. The case of harbor surveillance is a representative example since it opens challenging research problems, requiring processing information at different levels, from multi-sensor data to domain knowledge. Contextual information is essential for building a coherent and accurate surveillance concerned with security and safety events, to interpret sensed observations about a high number of different types of vessels appearing in diverse operations. The future work will focus in the formalization of the domain knowledge, extension of fusion framework with adaptation mechanisms, and multi-level fusion strategies to integrate sensor data and structured knowledge. 


\section{Acknowledgements}

This work was supported in part by Projects CICYT TIN2008-06742-C02-02/TSI, CICYT TEC2008-06732-C02-02/TEC and CAM CONTEXTS S2009/TIC-1485. A funded stay of Jesus Garcia in CMIF allowed the collaboration to define research strategies. The authors would like especially thank to Dr. Kessel, at NATO Undersea Research Centre, for helpful discussions on port traffic in the presented case of study.

\section{References}

1. Steinberg, A.N., Bowman, C.L.: Revisions to the JDL data fusion model. In: Liggins, M.E., Hall, D.L., Llinas, J. (eds.) Handbook of Multisensor Data Fusion. CRC Press, Boca Raton (2009)

2. Llinas, J.: A survey and analysis of frameworks and framework issues for information fusion applications. In: Graña Romay, M., Corchado, E., Garcia Sebastian, M.T. (eds.) HAIS 2010. LNCS, vol. 6076, pp. 14-23. Springer, Heidelberg (2010)

3. The Port La Spezia. Port Authority (2010), http://www.porto.laspezia.it (accessed November 2010)

4. On a Draft Roadmap towards establishing the Common Information Sharing Environment for the surveillance of the EU maritime domain. Commission of the European Communities, An Integrated Maritime, http: / / eur-lex . europa . eu / (accesed February 2011)

5. Benavoi, A., Chisci, L., Farina, A., Immediata, S., Timmoneri, L.: Knowledge-Based System for Multi-Target Tracking in a Littoral Environment. IEEE Trans. on Aerospace and Electronic Systems 42(N3), 1100-1119 (2006)

6. García, J., Guerrero, J.L., Luís, A., Molina, J.M.: Robust Sensor Fusion in Real Maritime Surveillance Scenarios. In: Proceedings of the 13th International Conference on Information Fusion (Fusion 2010), Edinburgh, UK (2010)

7. George, J., Crassidis, J.L., Singh, T.: Threat assessment using context-based tracking in a maritime environment Information Fusion. In: 12th International Conference on Issue Date: July 6-9, seattle, WA (2009)

8. Rhodes, B.J.: Knowledge Structure Discovery and Exploitation from Multi-Target Classifier Output. In: 7th International Conference on Information Fusion, Stockholm, Sweden (2004)

9. Gómez-Romero, J., Patricio, M.A., García, J., Molina, J.M.: Ontological representation of context knowledge for visual data fusion. In: Ontological representation of context knowledge for visual data fusion. 12th International Conference on Information Fusion, pp. $2136-2143$ (2009)

10. Perse, Matej, Ljubljana: A trajectory-based analysis of coordinated team activity in a basketball game. Journal Computer Vision and Image Understanding 113(5), 612-621 (2009) 